\title{
„Haltung provozieren" Ein Gespräch über Möglichkeiten der Kooperation zwischen universitären Lehrveranstaltungen und stadtpolitischen Initiativen
}

\author{
Anna Heilgemeir, Asli Varol, \\ Beatrice Termeer, Enrico Schönberg, \\ Dagmar Pelger und Paul Welch Guerra
}

Im Wintersemester 2014/15 fand am Institut für Architektur der TU Berlin eine außergewöhnliche Lehrkooperation statt. Unter dem Namen „Projekt X-Berg“ hat sich das „Fachgebiet für Städtebau und Urbanisierung“ CUD gemeinsam mit der Initiative „Stadt von Unten“, dem „Mietshäuser Syndikat“ und der „Kooperations- und Beratungsstelle für Umweltfragen“ KUBUS (ZEWK, TU Berlin) ein Semester lang mit den Potenzialen der Produktion von Stadt als Gemeingut beschäftigt.

In zwei Seminaren und einem Entwurfsstudio haben Studierende den üblichen Rahmen universitärer Lehre verlassen und sich mit Aktivist_innen und Mitgliedern der Initiativen ausgetauscht. Als gemeinsamer Bezugspunkt diente die Debatte um das umkämpfte sogenannte Dragonerareal in Kreuzberg. Der bundeseigenen Fläche droht die Privatisierung, wogegen sich unter anderem die Initiative „Stadt von Unten“ einsetzt.[1] Gleichzeitig bietet das Gelände eine Projektionsfläche für gemeingutbasierte Formen des Wohnens, des Arbeitens und Produzierens und des Zusammenlebens in der Stadt.

Mit Lehrbeauftragten und Teilnehmer_innen des Projektes haben wir über die Synergieeffekte und Erkenntnisgewinne, über das gesellschaftskritische Potenzial, aber auch über die Dilemmata eines Lehrformats gesprochen, in dem die Grenzen zwischen Lehre und Forschung, zwischen akademischer und aktivistischer Wissensproduktion und zwischen Seminardebatten und realen stadtpolitischen Kämpfen bewusst überschritten werden.

Für die Initiative „Stadt von Unten“ und das „Miethäuser Syndikat“ nahm Enrico Schönberg am Gespräch teil. Anna Heilgemeir ist freie Architektin unter anderem für das „Mietshäuser Syndikat“ und ebenfalls Mitglied von „Stadt von Unten“. Sie leitete als Lehrbeauftragte am FG CUD das Seminar „Selbstverwaltet Kommunal“. Studierende recherchierten historische und aktuelle Wohnungsbau- und soziale Infrastrukturprojekte, deren Finanzierung, Trägerschaften, Eigentums- und Organisationsmodelle als Grundlage dafür dienen sollten, über neue Formen der Kooperation zwischen Selbstverwaltung und öffentlicher Hand nachzudenken. Asli Varol, Architekturstudentin, und Beatrice Termeer, Urban Design Studentin, nahmen an diesem Seminar teil. Dagmar Pelger, freie Architektin und wissenschaftliche Mitarbeiterin 
am Institut „Entwerfen, Kunst und Theorie“ des KIT (Karlsruhe) bot als Lehrbeauftragte im Fachgebiet CUD das Seminar „Allmende als urbane Typologie“ an, das sich neben anderen Grundstücken in Kreuzberg ebenfalls auf das sogenannte Dragonerareal bezog. In engem Austausch mit den beiden anderen Seminaren erstellten Martin Murrenhoff, Jörg Stollmann und ihre Studierenden in dem Entwurfsstudio „Wohnen in Berlin 4 - Dragonerareal“ vier städtebauliche Entwürfe auf Grundlage zweier Entwicklungsszenarien. Ein Szenario basierte auf den Forderungen der Initiative „Stadt von Unten“ und ein zweites prognostizierte eine mögliche Kooperation zwischen „Mietshäuser Syndikat" und einer städtischen Wohnungsbaugesellschaft des Landes Berlin. Im Interview wird insbesondere auf die beiden Seminare Bezug genommen.[2] Das Interview führte Paul Welch Guerra.

Paul Welch Guerra (PWG): Von außen betrachtet ist die Struktur des „Projekt X-Bergs" nicht ganz einfach zu durchschauen. Wie ist die Konstellation zustande gekommen?

Anna Heilgemeir (AH): Wir sind von der Initiative Stadt von Unten aus auf den Lehrstuhl zugegangen und haben gefragt, ob sie nicht mal was zum Dragoner-Areal machen wollen. Auch weil wir gemerkt haben, dass wir als Initiative an unsere Grenzen kommen: Wir wollen ja dort ein Modellprojekt entwickeln, sind aber eigentlich die ganze Zeit mit dem Abwehrkampf gegen die Privatisierung beschäftigt. Dann kam die Idee auf, mit der Uni zusammenzuarbeiten. Interessanterweise hatten sie das Areal schon im Auge. Als Initiative waren wir vor allem daran interessiert, Recherchen über verschiedene vorbildhafte Projekte mit den Studierenden zusammen zu erarbeiten, um dann als Gesamtgruppe Interventionen zu entwickeln. Zunächst ging es uns also weniger darum, Wünsche zu erfragen, sondern darum, Wissen für einen Prozess der Stadtentwicklung von Unten bereit zu stellen. Diese Wissensproduktion haben wir dann gemeinsam durchlebt (lacht) und anschließend geplant, wie wir die Ergebnisse der Öffentlichkeit zugänglich machen.

PWG: Was ist das Besondere an dem Lehransatz?

$\boldsymbol{A H}$ : In unseren Seminaren versuchen wir beispielsweise über Haltung zu sprechen, bevor man Forschungsentscheidungen trifft. Ich finde auch, dass die Uni oder bestimmte Lehrstühle noch viel klarer eine Haltung beziehen könnten und dass es da gefühlt eine Angst davor gibt. Es bleibt oft diffus unter dem Stichwort „Akteursbestimmtes Planen“, anstatt mal klar zu sagen: „Es geht uns darum, dass die Armen nicht aus der Stadt rausfliegen.“

Dagmar Pelger (DP): Ein wichtiger Anspruch ist es auch, einen Austausch hinzubekommen. Seither entstanden drei weitere Kartierungs-Seminare zum urbanen Gemeingut, die sich mit Alltagspraktiken und alternativen Formen der Stadtproduktion beschäftigt haben. Das Fachgebiet hat auch eine Geschichte, wenn es darum geht, das Wissen von,draußen' an die Uni zu holen und umgekehrt. Zum Beispiel mit der „Akademie einer neuen Gropiusstadt“, an der ich auch beteiligt war. Da kristallisieren sich mit der 
Zeit methodologische Kniffe heraus, die man immer besser lernt anzuwenden. Ich kann mir gut vorstellen, dass man das unter den Studierenden auch zu schätzen weiß.

Asli Varol (AV): Als Architekturstudentin lebt man normalerweise in einer Gedankenwelt, man versteht sich untereinander. Man begründet seine Arbeit mit bestimmten Begriffen und bleibt dabei immer in einer akademischen Welt, ohne mal eine Initiative kennenzulernen oder zu wissen, wovon man eigentlich spricht. Es ist super wichtig, während der Planung mit NichtArchitekt_innen zu reden, die ein ganz anderes Wissen haben, um das zu vermeiden. Ich fand es gut, dass es im Seminar mal nicht nur um Gebäude und Städtebau ging, sondern andere Fragen im Vordergrund standen. Sich mit Finanzierungs-, Verwaltungs- und Organisationsstrukturen zu beschäftigen statt mit ästhetischen Erwägungen, ist eine Seltenheit. Wenn man nicht selbst den Anspruch hat und solche Projekte auswählt, kann man auch meilenweit daran vorbei studieren. Schon die aufwendige Darstellung von Entwürfen und Ergebnissen im Architekturstudium macht eine ernsthafte Beschäftigung mit Strukturfragen oft unmöglich.

Beatrice Termeer (BT): Ja, man arbeitet eigentlich immer auf Deadlines zu und bereitet Präsentation vor, die man beinahe täglich halten muss. Die Zeit, um mal einen Schritt zurück zu gehen und zu sagen: „Ich gucke mir das nochmal in Ruhe an und verwerfe es vielleicht auch komplett wieder", gibt es normalerweise nie. Auch die sozialen Aspekte des Entwurfs und des Austauschs mit Projekten außerhalb der Universität sind unter diesen Bedingungen meistens unmöglich.

Enrico Schönberg (ES): Die wissenschaftliche Welt hat oft ihren eigenen, teilweise inzestuösen Diskurs, in dem bestimmte Begriffe feststehen und definiert sind. Es ist ja auch Teil des wissenschaftlichen Arbeitens, Begriffe zu begründen. Eine Zusammenarbeit mit Initiativen, die sich in konkreten Auseinandersetzungen befinden, kann diese starren Begriffe ins Wanken bringen. Weil innerhalb von Konflikten die Infragestellung von Begriffen stattfindet. Weil Initiativen merken, sie werden in ihren Konflikten mit Erzählungen konfrontiert, die sie unterbuttern und ihre Forderungen unterminieren. Also bei uns war das zum Beispiel der Begriff der ,Bezahlbarkeit‘. Da wird schnell klar: Bezahlbarkeit ist nicht gleich Bezahlbarkeit. Eine Zusammenarbeit kann so Widersprüche innerhalb der wissenschaftlichen Debatten zum Vorschein bringen und etablierte Begriffe in Frage stellen.

$\boldsymbol{A H}$ : Spannend ist dabei nicht nur das Lehrformat, sondern auch, was für Netzwerke daraus entstehen. Wir bilden gefühlt einen kritischen Pool, auf den man zurückkommen kann. Auch wenn die Frage, was eigentlich ,kritisch heißt, noch nicht ganz beantwortet zu sein scheint.

PWG: Es geht euch also darum Planung beziehungsweise Architektur als etwas explizit Politisches zu verstehen?

$\boldsymbol{A H}$ : Ja genau. Das wird zu wenig gemacht, jedenfalls an der TU Berlin.[3] 
$\boldsymbol{A V}$ : Und das wirkt sich auch auf die Studierenden aus, die gar nicht mitbekommen, dass Architektur unbedingt etwas mit Politik zu tun hat. Und dass sie unbedingt noch andere Sachen tun sollten, das hängt alles zusammen. Das Angebot an der Universität, in diese Richtung zu gehen, statt nur zu studieren, ist wirklich sehr begrenzt. Oft geht ein Engagement in solchen Projekten auch damit einher, länger studieren zu müssen. Das können und wollen viele nicht.

DP: Ja, aber ich glaube, da kann man auch nur weitermachen und gegenarbeiten. Es gibt dieses Dogma, die Regelstudienzeit einzuhalten, das mag teilweise finanzielle Gründe haben. Aber ich hab den Eindruck, die ideologischen Gründe überwiegen. Die Studierenden, die ich kenne, stehen irgendwie in einer Bringschuld. Man kann nur dagegen arbeiten, und Engagement einfordern. Und dann muss man sich da als Studentin positionieren. Aus dem Allmende-Kartierungsseminar ist sogar eine Publikation mit vier Studierenden entstanden unter extrem großem Engagement.[4] So etwas kommt schon noch vor.

PWG: Eure Antworten zielen in erster Linie auf die Universität ab. Was hat das ganze Projekt den Initiativen gebracht?

$\boldsymbol{A H}$ : Wir haben eine vierwöchige Ausstellung mit den Ergebnissen im Foyer des Rathauses Friedrichshain-Kreuzberg gemacht, was ich schon aufgrund der Reichweite für einen ziemlich großen Erfolg halte.

BT: Die Ausstellung wurde am Eröffnungstag von unseren Aktionen im öffentlichen Raum begleitet. Es wurden Parolen wie „Wohnst du noch oder gentrifizierst du schon?" auf die Straße geschrieben. Darauf gab es Reaktionen. Das war spannend und hat Aufmerksamkeit erzeugt.

ES: Letztendlich ist es immer das Interesse unserer Initiative, unsere Inhalte in möglichst viele unerreichte Räume und Gruppen hineinzutragen. Bei der Eröffnung waren unheimlich viele Leute und alle haben mitgekriegt: Es gibt mal abgesehen von den präsentierten Ergebnissen eine Auseinandersetzung um das Areal. Die Ergebnisse zeigen darüber hinaus, dass wir nicht nur gegen Privatisierung sind, sondern an konkreten Inhalten arbeiten, dass unsere Inhalte wissenschaftlich fundiert sind, und dass wir nicht die NIMBYs[5] sind, die alles nicht wollen (alle lachen). Hier entsteht ein wirkmächtiges Argument rein aus der Kooperation. Die Ergebnisse haben wir auch im Rahmen eines Nachbarschaftsfestes und auf einer Konferenz nochmal vorgestellt.

$\boldsymbol{A H}$ : Für die Initiativen war es gut, dass ihre Ideen plötzlich auf Papier sichtbar wurden. Es war auch ein Experiment zur Zusammenarbeit mit universitären Lehrveranstaltungen. Was können diese sogenannten Expert_innen überhaupt? Und wie kann man kollaborieren? Das wäre auch ein Ziel: dass dieses Wissen innerhalb der Initiativen weitergetragen wird. Damit die Initiativen merken: Ich muss nicht immer nur Anfragen beantworten, ich kann auch aktiv auf die Universität zugehen und fragen. 
ES: Andere Akteure machen auch nichts anderes, die Wirtschaft nimmt sich die Wissenschaft auch gerne zur Hand. Sie schafft sich Studien, Aufträge et cetera, die einen neutraleren, objektiveren Standpunkt behaupten sollen. Objektiv sind sie natürlich auch nicht unbedingt, aber die Behauptung hilft, um politische Ziele durchzusetzen. Wenn wir selber an dieser Möglichkeit arbeiten und sie nutzen, finde ich das jedenfalls gut. Zum Beispiel nutzen Initiativen immer wieder die Studien von Wissenschaftler_innen zum Wohnungsmarkt, etwa um zu zeigen, dass es nicht genug bezahlbaren Wohnraum gibt. Und dann stellt sich natürlich die Frage: Wie kann man so etwas in einem Verhältnis von Initiative, Lernenden und Lehrenden reproduzieren?

PWG: War das alles so harmonisch, wie es rüberkommt?

$\boldsymbol{A H}$ : Naja, es gibt innerhalb der Initiative auch eine akademiekritische Fraktion. Sobald es offizieller wird, wird es einigen dann auch schnell mal unheimlich. Also, wollen wir überhaupt was im Rathaus machen? Wollen wir anfragen oder wollen wir den Raum einfach besetzten? Die Kooperation mit der Universität hat Letzteres ja eher unmöglich gemacht. Es gab sicherlich unterschiedliche Meinungen dazu, aber es war auch klar, dass die Öffentlichkeit, die dadurch generiert wird, einfach wichtiger ist als die Vorbehalte. Ganz harmonisch war es jedenfalls nicht. Und die städtebaulichen Entwürfe wurden teilweise auch durchaus kritisch gesehen. In den Entwürfen war eine relativ hohe Bebauungsdichte vorgesehen und Hochhäuser provozieren in Kreuzberg natürlich Widerspruch. Da sehe ich vielleicht auch ein bisschen eine verlorene Chance. Mit mehr Zeit hätten wir diese Gestaltungsfragen besser ausdiskutieren können.

Innerhalb des Lehrstuhles gab es dann noch den kritischen Moment, als die Initiative einen Info-Tisch in das Rathaus stellen wollte. Es wurde in Frage gestellt, ob es überhaupt geht, bei einer Ausstellung der Universität einen Info-Tisch von „Stadt von Unten“ zu präsentieren, über dessen Inhalte wir dann nicht mitentscheiden. Da musste ich dann versichern, dass der Tisch als Exponat der Initiative erkennbar bleibt und sich von den kooperativ entstandenen Ergebnissen absetzt. Da wir ein Gestaltungsstudiengang sind, gab es auch Bedenken, ob das formal passt. Wir haben das dann in verschiedenen kleinen Gesprächen viel auf Vertrauensbasis abgestimmt. Und dann gab es natürlich auch noch Leute aus der Initiative, die eigenmächtig Plakate aufgehängt haben.

ES: Was feststeht: Es hat eine große Wirkmächtigkeit, wenn man solche Kooperationen eingeht, und das kann einen auch überfahren, wenn die wissenschaftliche Institution, mit der man zusammenarbeitet, anfängt etwas zu produzieren mit der Behauptung, man hätte zusammengearbeitet.

\section{PWG: Also eine Art der Vereinnahmung?}

ES: Ja. Also dieses „Wir haben ja zusammengearbeitet“. Und dann wird etwas produziert, was von Anderen wieder ganz anders verwendet wird, aber man ist dann gefangen, weil es heißt „Ihr wart ja mit dabei bei der Entwicklung“. Auch bei den Entwurfsprojekten, bei denen es ganz konkret wird, kann so 
etwas problematisch werden. Wie werden die Entwürfe verwendet? Manche Studierende wären umgekehrt sicher ganz froh wenn ihre Ergebnisse nicht in der Schublade des Archivs verschwinden, sondern durch eine Initiative verwendet werden. Es kann dann aber wiederum auch passieren, dass die Initiative die Ergebnisse quasi enteignet und Sachen damit macht, die nicht im Sinne des Forschenden waren.

$\boldsymbol{A H}$ : Beim Seminar haben wir uns darüber ja verständigt. Wir haben ein Gespräch darüber gehabt, ob das ok ist, dass Stadt von Unten die Ergebnisse über das Seminar hinaus weiterverwenden und -entwickeln kann.

$\boldsymbol{E S}$ : Das ,Ob“ ist aber nur ein Teil der Frage. Das ,Wie‘ ist damit noch nicht beantwortet. Und über dieses ,Wie‘ könnten Lehrende auch die Kontrolle verlieren in dem Moment. Das ,Wie‘ und,Wofür‘ kann man nur klären, wenn man auch eine kontinuierliche Zusammenarbeit hat. Und das ist mein Punkt: Wir haben zwar eine Kooperation, aber in der Perspektive organisieren wir uns nicht zusammen und wir haben keine längere Zusammenarbeit, in deren Rahmen man gemeinsam darüber entscheidet, was man mit dem produzierten Wissen anstellt.

PWG: Stichwort Perspektive: Was für ein gesellschaftskritisches Potenzial seht ihr in solchen Lehr- und Forschungsformaten?

DP: Ein wichtiges Potenzial liegt sicherlich darin, eine Verschiebung der gesamten Konstellation zu erreichen. Also dass man versucht, über die Kooperation und andere Hebel und methodische Herangehensweisen die Studierenden und auch sich selbst aus der Uniperspektive gedacht in eine andere Position zu bringen, die gewöhnliche Bezüge zueinander aufzuheben: Eine Haltung zu provozieren. Es geht darum rauszukommen aus dieser klassischen professionellen Sicht auf diese Disziplin ,Architektur/Planung und ihre Werkzeuge, die man da lernt und lehrt. Klar vermitteln wir auch ganz klassisch Werkzeuge. Aber eben auch, wie diese ganz anders eingesetzt werden können. Das fängt im Grunde schon im ganz Kleinen bei der Art der Aufgabenstellung für einen Entwurf an: Welches Programm gebe ich vor? Hinterfrage ich das Grundstück? Hinterfrage ich das Seminarthema? Und im Großen heißt das dann, die Aufgabe, die man sich gesetzt hat, mit dem echten Leben da draußen zu konfrontieren.

$\boldsymbol{A H}$ : Ein Potenzial besteht sicherlich auch in der Möglichkeit, die Rolle der Planer_innen anders zu definieren, als das herkömmlich geschieht. Das bedeutet, sich mehr Zeit zu nehmen, die Strukturen dahinter zu hinterfragen. Es ist mir ein Anliegen zu vermitteln, dass Architekt_innen darüber reflektieren, inwieweit sie ihre Auftraggeber_innen selbst aussuchen können und sich nicht einfach vom Markt bestimmen lassen. Man kann sich Auftraggeber_innen danach aussuchen, inwieweit sie ein ,kritisches' beziehungsweise veränderndes Potenzial haben, Räume zu bauen. Um das zu vermitteln, muss man die Rahmenbedingungen verändern. Viele Studierende verlieren schon während ihres Studiums die Vorstellungskraft, wie ein Arbeitsalltag anders aussehen könnte als dass man in einem Büro arbeitet und weisungsgebunden 
irgendwelche Sachen plant, die man eigentlich nicht gut findet. Dafür ist die Kooperation oder sogar Kollaboration mit stadtpolitischen Akteuren ein total wichtiger Schlüssel. Sätze wie „Wow, was ihr als Initiative alles geschafft habt“ oder "Ich dachte, dass in solchen Projekten nur Hippies wohnen“ zeigen, dass eine Verschiebung des ,Ernstnehmens ' von Alternativen stattfindet. Also von Projekten, die nicht nur, alternativ 'sein wollen, sondern die wirklich machbar sind. Dazu gehört auch zu verstehen, dass solche Projekte nicht im Alleingang zu realisieren sind. Es gibt so viele Planer_innen, die im Alleingang versuchen, irgendwelche Firmen aufzuziehen, wo sie dann Projektberatung für ,Co-Housing-Lalala-Alternative-Projekte' machen. Das ist meiner Meinung nach totaler Schwachsinn. Das kann vielleicht ökonomisch funktionieren, aber das hat nicht dieses gesellschafts-verändernde Potenzial. Meiner Meinung nach ist Stadt eine Gemeinschafts-Produktion. Und das ist der Grund dafür, dass diese Seminare so gut sind, weil man hier genau das eben kollektiv lernt.

PWG: Vielen Dank für das spannende Gespräch.

Das Gespräch führte Paul Welch Guerra.

\section{Endnoten}

[1] Für mehr Informationen zum Gelände, dem Privatisierungsprozess und der Initiative: www.stadtvonunten.de.

[2] Für mehr Informationen zu den Lehrveranstaltungen: http://www.cud.tu-berlin.de/ topics/spatial-commons-2/

[3] Zur Tradition einer politischen Betrachtung von Architektur und Planung an der TU Berlin und anderswo: Nina Gribat, Matthias Görlich und Philipp Misselwitz (im Erscheinen 2016): Vergessene Schulen: Architekturlehre zwischen Reform und Revolte um 1968. Leipzig: Spector Books.

[4] Dagmar Pelger, Anita Kaspar, Jörg Stollmann (Hrsg.): Spatial Commons. Städtische Freiräume als Ressource. Berlin 2016; kostenloser Download: http://dx.doi.org/10.14279/ depositonce-5075.

[5] NIMBY = not in my backyard 
